\title{
ESDA2006-95308
}

\section{A CO-ROBOTIC POSITIONING DEVICE FOR CARRYING SURGICAL END-EFFECTORS}

\author{
Silvia Frumento, Rinaldo C. Michelini \\ University of Genova, PMARLab, DIMEC \\ Via all'Opera Pia, 15/A 16145 Genova, ITALY \\ Tel: +39 0103532837, Fax: +390103532298 \\ E-mail: michelinir1@asme.org.
}

\author{
Rainer Konietschke, Ulrich Hagn, \\ Tobias Ortmaier, Gerd Hirzinger \\ German Aerospace Center (DLR), \\ Institute of Robotics and Mechatronics \\ D-82230 Weßling, GERMANY
}

\begin{abstract}
The development of a remotely operated, Co-Robotic Positioning Device (CRPD) for instrumental backing and optimal base position to robotic arms in tele-surgery is discussed. To optimise the setting of robotic operating rooms (ROR) by reducing the structures' size around the patient and by selecting task-driven layouts, the design of a hanging servocarrier coming from the ceiling is chosen, rather than a device located on the floor. The present study prospects a split-duty approach, distinguishing the Co-Robotic Positioning Device, CRPD, from the front-end effectors, each subsystem hierarchically controlled by remote location, in keeping with optimal protocols. The attention is focused on the slave-carrier, to establish an optimal design of the CRPD, based on the characteristics of robotic effectors and the surgical task. The CRPD is conceived to support (up to four) robotic effectors, each one equipped with proper tools (endoscope, scalpels, scissors, suture needles, etc.). The CRPD, actually, by optimally positioning the robotic arms, avoids the need of manual deployment, in current setups often necessary to avoid singularities or collisions. The Automatic Changing Device for Surgical Tools, ACD-ST, is another significant device of the conceived slave-carrier. It allows the tele-operating surgeon to change the tools (scalpels, scissors, etc.) by a direct command from his console. Example applications aim at ticklish endoscopic/tomic operations that require high accuracy with low involved forces such as cardio-thoracic-surgery, abdominal surgery, spine-surgery, microsurgery (neurosurgery, handsurgery, ophthalmic-surgery, ear-nose-throat surgery), say, the typical domains of MIRS, where robotic surgery is quickly expanding.
\end{abstract}

\section{INTRODUCTION}

Robotics has pervasive spreading in everyday life, as it provides options highly enhancing the access to current functions with wider capabilities and higher performance. This general axiom is valid for surgery, as well, which requires the on-site presence of agents with the twofold bent "recognitionand-intervention" in view to accomplish finalised tasks, with intelligence of the on-progress operations. Such premises, actually, are starting demand in instrumental robotics, where effectiveness is given by duty-driven solutions, suitably balancing material-and-information handling. The robotics achievements in medicine [1], thereafter, follow complementary tracks, encompassing:

- Task acknowledgment, by process-data acquisition and diagnostic features assessment;

- $\quad$ Task execution, by operation sequencing and adapting by work-progression monitoring;

under the surgeon's steering by direct, either, remote supervision and control. The robotic instrumental aids are included to improve and to widen information and functional bases, with noteworthy up-grading of merely human capabilities.

The upgrading in robotics is technology-dependent, with traditional role played by mechatronics and ICT, and with still undisclosed possibilities offered by the nano-technologies. Basically, surgery, up to now, moves within the scale of visual and tactile discrimination; standard in-body interventions required large openings, deep to create an operation foreground accessed by sight and by hands; recovery is highly affected by these side issues, in spite of the impressive advances of modern medicine. 
Robot-assisted surgery is said to provide turns similar to machine-tools manufacture after handicraft. In tele-operated robotically assisted surgery, artificial effectors are made available, with miniature size, even below standard human handling scale. The operation theatre is accessed directly by percutaneous needles or, through trocars, by articulated rigs carrying tools; the action is monitored by local miniature cameras or through CAT images of externally located devices. The surgeon needs to remotely control, by master-slave command of the carried tools, according to different operation protocols, which might, eventually, transfer in charge to the robotic devices the autonomous accomplishment of preset subtasks.

The robots, according to the sketched frame, are the enabling support of the minimally invasive surgery, MIS, the technique aiming at localised interventions, with negligible side effects. Today, pioneering steps only are moved, as compared to potentials, since robotic aids are under development and reliable protocols need to be established based on properly acknowledged experience, leading to the emerging domains of minimally invasive robotic surgery, MIRS. In these frames, to develop a remotely operated co-robotic positioning device, CRPD, for instrumental backing and optimal base position for robotic arms in tele-surgery, is discussed. In confined reality, such as the Operating Room, OR, different kinds of surgery require a wide range of equipment and, in the case of the Robotic Operating Room, ROR, technical apparatuses take the challenging role of covering wide spectra of functions, especially out of man tactical and optical perception. The effectiveness will result from exploring the robot technologies as enabling options in front of tasks, which should be performed, but could only be achieved with high invasiveness by direct surgeons' intervention. The discussion develops in the paper with an overview on technologies, as starting hints to specify the instrumental CRPD aid.

\section{OPERATIONS IN SURGERY ROBOTICS}

Moving on along the recalled comments, the robot opportunities are typical technology-driven aids, which need shared acceptation and habit appropriateness to enter into common practice. The instrumental assistance requires revising assessed habits in existing protocols, looking after a different surgeons' involvement, little by little less urged to reach the highest skill in manual dexterity and progressively required to defer this to front-end autonomic effectors and to co-ordinate multiple tasks, simultaneously done by instrumental aids.

Minimally invasive robot surgery, MIRS, thereafter, is believed to move towards developing specialised co-robotic devices, to be integrated, based on the selected functions, in the operation work-plan, in keeping with the capabilities assigned to the individual fixture. Basically, instrumental robots characterise because of [2]:

- Function bent presetting;

- Task programming and updating;

- Duty progress monitoring;

- Surroundings overseeing;

- Autonomy management within allowed specifications.
These robot aids are required to work inside properly structured bounds; however, the environment it is interacting with belongs to alive matter, and might quickly turn to dramatically different situations, thus:

- Changes need to be acknowledged;

- Emergency plans and safe recovery shall autonomously start;

- All information ought to be displayed to the on-duty surgeon, with, subsequent steps suggestions.

The proper setting of autonomy limitations is a subtle question, and, in any case, trimming to individual whims or complete overriding needs to be possible.

MIRS provides technology-driven winning opportunities, mainly, in two domains:

- Information infrastructures: data acquisition, handling, transmission, validation, processing, etc. are continuously expanding options supported by the ICT, and effective new computer tools ceaselessly appear to support remote supervision and control; tele-medicine is a fully acknowledged technology, while tele-surgery has already experienced noteworthy accomplishments [3-5].

- Effectors: specialised tools and fixtures are the most challenging research incumbents, possibly, today, too much tied up with human handling scale; in the future: the surgeons shall continue to deal with standard sizes; the inner-body interface will timely evolve toward micro- and nano-apparatuses, as soon as effective new solutions are conceived and made available $[6,7]$.

The investigation addresses this second domain, looking at intrinsic effectiveness layouts, and attention on the merging between information and material flows is the characterising feature. The MIRS, actually, is a critical upgrading of MIS, by removing the instrument-surgeon's direct link and related accessibility bounds, through trocars or percutaneous paths, and by enabling remote control by master-slave techniques or similar means. The MIRS setups allow:

- Tactile feedback to appreciate touched items compliance;

- Kinaesthetic restitution to fully control the grasping/handling forces;

- 3D visualisation to support correct hand-eye coordination;

- 6 DoF manipulability to supply full dexterity for inbody actions.

The four functional requests need to integrate technical frames, built on:

- End-effectors mobility redundancy;

- Intelligence for autonomy management;

- Operation reliability and intrinsic safety;

- On-process diagnostics and self-recovery.

These achievements basically exploit virtual reality restitution and behavioural emulation: realistic imaging, intuitive/ergonomic interfaces, duty-cycle assisted progression, automatic monitoring and completion of preset tasks, etc.; new intervention techniques and protocols will develop, depending 
on the technologies timely available. A survey on the field shall necessarily start from the technical constraints, as these are the intriguing contributions to foster innovation.

\subsection{The Split-Duty Concept}

Reliability and safety are MIRS preliminary demands, as:

- Interventions apply on human beings;

- Robotic rigs are in contact with patients (and staff);

- The responsible operators (surgeons, etc.) are not robot-experts.

The instrumental aids, thereafter, need to grant complete transparency to the supplied function, with no bias of mechanical couplings, information handling, reconfiguration procedures, etc., that, possibly, occur. To that scope, a surgical device has always to comply with overall requirements: no uncontrolled motions; bounded output forces/displacements; self-recovery end-effectors; constant surgeon's overseeing. The overall requirements address:

- $\quad$ Redundancy, in mobility, sensors and control;

- Recovery, in function, actuation and intelligence.

Advanced robotic architecture and operation strategies could apply, such as the split-duty approach. The concept characterises, as for the hardware, by Fig. 1:

- the auxiliary rigged frame, to grant correct positioning and feeding of the MIRS setup;

- the front-end effectors, to accomplish the in-body access of tools and cameras.

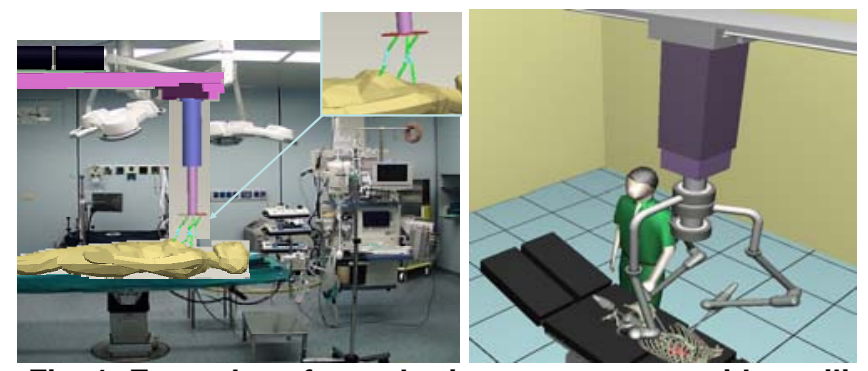

Fig. 1: Examples of co-robotic arrangements with auxiliary carrier and carried end-effectors

As for the software, each subsystem is hierarchically controlled by a remote location, in view of the chosen protocols, while the interfaced operators receive subsets of information at the selected degree of immersion, with suggestions of consistent (vs. not advisable) actions.

The split-duty approach can expand at different levels of sophistication. In general, it follows the concept of functional allocation and structural modularity to manage redundancy and recovery and to enhance reliability and grant safety. The final layout aims at provisions duplication by preserving leanness. The example arm-selection process can be used for explanatory purpose [8]. A robot conventionally characterises by a (serial or parallel) arm (and pertinent tools), so that the tip work-space is acknowledged and ruled by means of the actuation joint-space. Now, the in-body work-space, accessed by locally constrained paths (e.g., trocars), is encumbered with obstacles (say, connecting tissues) and blocked up with forbidden zones (say, vital organs). The initial design request is: how many actuated joints assure $6 \mathrm{DoF}$ at the tip? Conventional analyses show how to oblige an arm member to slide along a space point (trocar location), with fixed slope (trocar inclination); alternatively, with sensors on the trocar, the feedback acts on the arm mobility, to keep fixed position and slope. It is more questionable to deal with in-body restraints, as the vital organs modify shape and size and the intervention point needs to adapt to changing conditions; the choice of an optimal arm path is a possible guess to start with; then, resort to local displacement or pressure sensors requires critical observation gauging and path planning steps, to face uncertainty.

By the split-duty approach, the arm-selection process aims at, separately, programming the carriers and the effectors. Several competing layouts can be considered; in general:

- Carriers are arms, with distal rigs held, controlled in position and attitude, near the patient's body;

- Effectors are tools for in-body interventions, assuring convenient insertion and manipulation.

A hierarchic controller is expected to manage the all. Each carrier position and attitude are set and tuned, depending on the effectors' requests, along with duties progression. The splitduty aims at optimality, considering as primary instance the effectors' requests (based on the fusion of sensor data and visual checks). The overall redundancy is turned to satisfy separate requirements, depending on the timely addressed task assignment. Force feedback from the operation interface (trocars and effectors), friction compensation, shape updating for proper engagement, etc. are added opportunities, offered to the surgeons as the case arises, while the routine progression deploys with continuity upon their consent, following standard protocols.

\section{CO-ROBOTICS TECHNOLOGIES}

The split-duty approach, typically, requires layout redundancy, as well. Then, computer-assisted surgery expands as technology-driven option, to encompass:

- Passive aids: navigation and aiming devices, enhanced restitution displays, etc.;

- Support aids: action guided interventions, based on selected strategies, finally enabled by the surgeon;

- Autonomic aids: task sequences performed by the robot, under the watchful eye of the surgeon.

The information infrastructures basically supply passive aids and co-operate to the feasibility of autonomous or cooperating aids. The execution effectors aim at replacing the tangible contributions of human operators with benefits in accuracy, delicacy, dexterity, efficiency, safety, size, versatility, etc. once the instrumental properties are optimised (and anthropocentric limitations are overrun).

The focus on execution effectors draws attention to the quick evolution in the nano-technologies; they will, probably, deeply modify these instrumental aids. A survey to summarise some available means is, therefore, useful mainly to specify underlying concepts and functional modules in view of future issues. The split-duty approach quite naturally leads to modularity. The auxiliary handling frame can readily exploit 
standard arms, up to a rigged-out carrier, holding the special end-effectors and related feeding and controlling interfaces. The design of a MIRS environment, thus, typically leads to jointly develop the servo-carrier modules and the (dexterous) devices, with front-end in-body tools. A few remarks help explaining the advantages.

The first generation of surgical robotic systems, e.g. the cardiothoracic surgery daVinci ${ }^{\mathrm{TM}}$ Robot, have the drawback of huge overall size, with path hindrances and collision risk concerning the system itself or other equipment in the operating room. It is desirable to optimise the setting, reducing the structures' size around the patient and looking after task-driven layouts. This results, for instance, in hanging co-robotic carriers, coming from the ceiling, rather than from the floor.

Moving from effective CRPD aids, the further characterising modules of the end-effectors include:

- The carrier plug-in block, for latching/unlatching actions and power/control lines connection;

- The holding blocks, to provide correct insertion in the patient's body, as for location and attitude;

- The in-body blocks, to grant proper reach to the operation theatre through a safe path.

Only in-body blocks request sterile and miniature components; a safe and fast (bayonet, etc.) coupling could be devised, to link/disconnect them from the other blocks. To gain dexterity inside the patient body, the simplest setups aim at a needle, through a percutaneous notch, suitably directed to the entry location and along the best path slant. Slightly more sophisticated arrangements deal with thin rods [9], inserted through trocars; at the front, a 2 DoF wrist and 1 DoF tool are driven, most of the time, by cables actuated by off-body motors. The resort to articulated probes has been considered, often for endoscopy and specialised (laparatomic, gynaecologic, etc.) interventions; the probe is built with a series of blocks, with slightly bigger diameter, and actuation deferred to motors carried within each block. In these cases, the independently controlled supporting slave carrier, mainly, has the task to rationalise the intervention, splitting the protocols for the effectors positioning, from the tips engagement.

\subsection{Reference Technologies: Layouts and Setting}

The split-duty approach and remotely operated co-robotic positioning device, CRPD, dramatically increases intervention effectiveness, when multiple end-effectors are required. In these situations, Fig. 1, the co-robotic carrier is, mainly, a passive aid, bringing the effectors nearby the operation theatre, so that their engagement takes place without interference risks. Basically, two approaches could be explored:

- Articulated miniature effectors, devised to reach the operation theatre along almost entirely in-body paths; the carrier lay some centimetre away from the patient's body, and the effectors are powered only to accomplish the localised tasks, while the carrier modifies the attitude for location trimming; Fig. 2a shows an example setup, namely the miniature probe, providing a 7 DoF effector, with resort to $(10 \mathrm{~mm}$ diameter) powered-segments [10], assuring tip thrust of about $40 \mathrm{~N}$ and torque of $8 \mathrm{mNm}$ every module, with limited reach due to the small size of the overall mechanism (length $\cong 150 \mathrm{~mm}$ in the case of 3 modules);

- Modular articulated arms, with appropriate versatility to reach alternative operation theatres along outerbody paths; the carrier is located sufficiently apart, to allow proper arms deployment, possibly, by switching to different positions/attitudes, whenever requested by the protocols. Fig. 2b) shows the DLR medical arm, providing a 7 DoF effector, with resort to controlled members, assuring a maximal payload of $3 \mathrm{~kg}$ throughout the whole workspace [11].
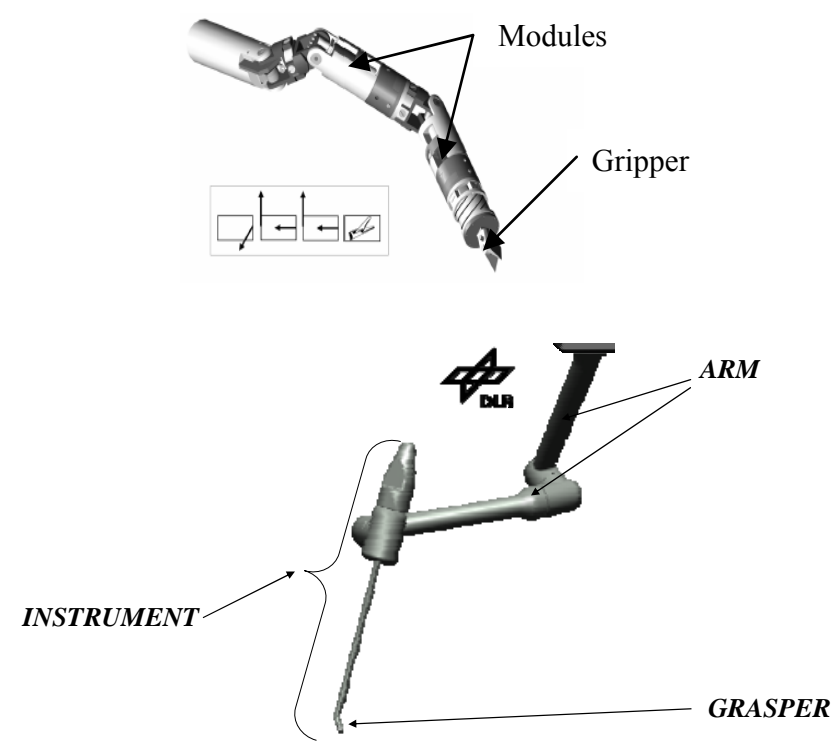

b)

Fig. 2: Example robotic effectors: a) miniature probe; b) compact arm.

The attention is focused on the slave-carrier, to establish an optimal design of the CRPD, based on the characteristics of robotic effectors and surgical tasks.

The CRPD is conceived to support (up to four) robotic effectors, equipped with proper tools (endoscope, scalpels, scissors, suture needles, etc.). Tasks, typically, refer to minimally invasive robotic surgery, MIRS: before operation begins, the CRPD, starting from a remote location compared to the operating table, positions itself automatically, carrying the robotic effectors bases at the desired and precise position generated in the preoperative planning (see e.g. [12] for further details). Then, the effectors reach the patient and the tips are inserted in the patient's body, through trocars at well defined locations, according to the scheduled intervention. As the surgeon starts operating, the CRPD is fixed in its standing position, and the effectors only are controlled, to accomplish the planned duties, unless the preoperative planning includes intermediate displacements, or the surgeon, himself, looks for better locations during the intervention. 
As regards the OR's investigation, several elements have to be considered:

- Compatibility with every OR

- Interference avoidance with existing equipment or staff in the OR

- Small size

- Fast removal from the patient in case of complications

- Easy storage when not in use

Additionally, the following important requirements have to be met:

- Compatibility with the ACD-ST

- Support of the workflow

- Easy robot-human interface

- Safety issues (for the patient and the surgical staff)

- Easy cleaning and sterility requirements compatible

- Easy assembling/ disassembling/maintenance/ repair.

The exploration of the OR setup during a heart-surgery operation, when up to 9 staff members are required as well as huge equipment (like the heart-lung-machine), leads to the demand for a design that leaves as much space as possible to the OR staff. Furthermore, in case complications occur, the CRPD has to be removable rapidly from the patient, taking a good storage position to allow the surgeons to quickly reach the patient for direct treatment.

\subsection{Reference Technologies: Carrier and Plans}

The co-robotic carrier could suitably be attached to the ceiling as seen in Fig. 3, travelling along linear guides and with a lifting tower for setting the vertical location, where the operating arms are placed, with independent pivot, bending and twist commands. With the miniature probes (see Fig. 4), proper guiding rigs, or dispensers, are needed, suitably to be located in parallel, to assure balanced minimal paths, to the co-operating effectors. Of course, different other carrier's arrangements could be devised, and the sketched examples are given for explanatory purpose only. It should be pointed out that the two considered effectors, the DLR arm and the miniature probe, represent typical solutions of a high performance general purpose surgical robot, either, highly specialised local effector, today under investigation for advanced interventions. Noteworthy cases requiring ticklish endoscopic/tomic operations, with high accuracy and reliability, with not considerable involved forces, are more and more entering in surgeons' practice, insofar as the diagnostic tools reach better discrimination of the disease. The fields of interventions cover microsurgery, say: neurosurgery, ophthalmic-surgery, ear-nosethroat surgery, hand-surgery, etc., and, in general, the domains of MIRS, where robotic is providing alternatives out of the standard human hand and eye reach. In other fields, cardiothoracic-surgery, abdominal surgery, spine-surgery, etc., considerable forces/torques are required, and robotic expansion needs necessarily look after appropriate solutions, that, generally, explore more anthropomorphic approaches.

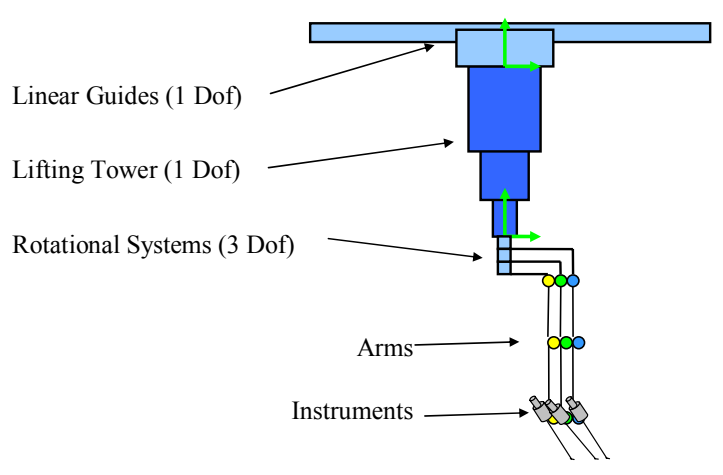

Fig. 3: Example of a telescopic tower for the carrier. a)

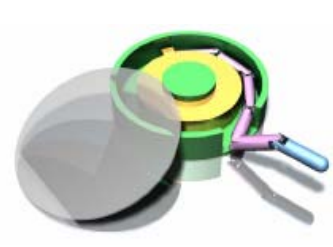

b)

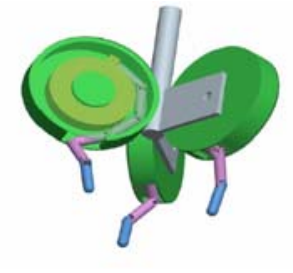

Fig. 4: Example of a miniature probes dispenser and of the parallel arrangement.

In the different domains, the reference technology develops, according to even approaches, properly detailed operation plans and related protocols. The complexity of the interventions, most of the times, cannot be fully accomplished by the co-operation between the same two effectors, and multiple tools and/or fixtures need be sequentially brought foreground. This is easily done, if the co-robotic carrier is endowed with an Automatic Changing Device for Surgical Tools, ACD-ST, namely, rigged slots for the quick insertion of the appropriate arm or probe dispenser, whenever requested. This allows the tele-operating surgeon to change the tools (scalpels, scissors, etc.) by a direct command from his console, on condition that the properly outfitted effector is placed into addressable slots of the slave carrier.

\section{CONCEPT DESIGN OF THE CO-ROBOTIC CARRIER}

The bird eye survey of the two example technologies shows how split-duty planning, enabled by co-robotic arrangements, expands versatility and reliability exploiting functional redundancy. Benefits are reached by:

- The choice of task-driven operation protocols, so that the handling outfit is optimally tailored to the preset planning, beforehand adapting the hardware instrumental aids, to the intervention schedules;

- The choice of modular-arranged resource architectures, so that the preoperative planning is readily mapped into convenient handling outfits and effective operation sequences, by proper duty allotment.

The concept design of the co-robotic carrier follows these requests. Introductory hints are hereafter given, only retaining the ceiling hanging-down configuration, which permits 
solutions in good consistency with the existing operating rooms.

\subsection{Choice of operation protocols}

Preoperative planning distinguishes two phases, say, handling outfit laying and agenda schedule; the twos require previous acknowledgement of patient and intervention data. Today, detailed diagnoses may be based on accurate specific tomography data, recorded and segmented to infer 3D models of the anatomical structures of interest. The surgeon plans the intervention on the basis of the virtual patient model; he selects the organs and parts, which must be reachable by the instruments and visible by the endoscopes, and makes automatic inquiry of the optimal arms and effectors locations; on-duty configurations; approach and engagement paths; with warnings as for critical nature, as for solutions robustness. The intervention is simulated in a virtual environment, to explore reliability. In case of faults, the planning is possibly modified and the optimisation is redone. Then, the preoperatively data is transferred into the OR. The first step in doing this is to map the virtual environment to the real anatomy of the patient; thereafter, the arms and effectors are positioned according to the preoperative plan. The second step deals with on-duty constraints. In MIRS, e.g., beating heart surgery motion compensation [13] is a highly desired option: the robot cancels out the heart motion, such that the relative pose between heart surface and centre point of the surgical instrument remains constant. The surgeon, thus, works on a virtually stabilized heart, as he was used to in the on-pump surgery, where the heart-lung machine is used to sustain the circulation and the heart does not move. This achievement, it is worth noting, is still current research subject; the off-setting of the heart motion is achieved by stabilizers, but residual tissue motion makes surgery still difficult and time consuming, unless duty trimming adds.

This stresses the importance of communication and cooperation between devices for agenda scheduling in the OR to obtain proper fixture guides during the intervention. It is important to underline that, on the base of the followed splitduty approach, the robot is conceived to be composed of multiple components that are designed, moved and controlled separately. In these terms, it is sufficient to assign certain features like e.g. portability only to those subsystems that have to be serviced and sterilized continuously and that can thus be mounted in the OR at short notice before the intervention.

The split-duty method enables, in our case, a transparent structure of the complex intelligent robotic system made up of submechanisms that can work independently or interact, depending on the phase of the intervention. A robotically assisted medical intervention can be structured in three stages:

- Positioning of the robots (before the operation starts); split in two subtasks: platform location and endeffectors deployment;

- Intervention;

- Removal of the robots from the patient; again split in the two backward subtasks as previously mentioned.
Each subsystem works according to specific protocols that establish when it is scheduled to work alone or to co-operate with the other, on passive or active conditions. Table 1 summarizes the devices' function during the three stages. Each stage involves at the same time different subsystems: the CRPD, the Robotic Arms (with the respective tools) and the ACD-ST. The presence of different devices leads to divide each stage in two different steps reflecting which device is moving.

Once the patient is stabilized and monitored, the operation begins with the insertion of the trocars, where surgical tools and endoscope shall insert. The CRPD then positions itself according to the preoperative planning, followed by the placement of the Arms and their engagement through the trocars. As Table 1 shows, the CRPD is the only active device during Step 1, while in Step 2 the CRPD does not move and hence it assumes a semi-active role, defined as to support and grant high stability to the arms during the tools' introduction in the patient body. Then, the surgeon starts the operation from his console and controls the surgical tools and the endoscope, to perform the intervention. Should the surgeon need a different tool, he applies for the automatic change procedure carried out by the ACD-ST: the effector removes itself from the patient body and reaches the CRPD; then the ACD-ST provides the new instrument and accomplishes the change. During the operation, the CRPD supports and grants stability to the effectors, to provide the expected accuracy (a tolerance of 1 $\mathrm{mm}$ is acceptable for the CRPD and the ACD-ST); as the case arises, the CRPD modifies position and/or attitude, depending on the agenda schedules and/or the surgeon decisions.

When the intervention is completed, the effectors move back from the patient; steam-sterilizable tools, in patient contact are manually separated from the spray-sterilizing parts, not in patient contact. During the last step, the CRPD carries the arms in a remote location where the effectors are stored and serviced.

The split duty approach that distinguishes each system's function does not exclude the interaction among the subsystems, but it instead increases the possibility to easily control each device in order to facilitate the communication protocols. In particular, the co-operation is established during the intervention itself (Step 3), when the devices are activated at the same time. 
Table 1: The system's split duty approach.

\begin{tabular}{|l|c|c|c|c|}
\hline \multicolumn{2}{|c|}{} & \multicolumn{2}{c|}{ SUBSYSTEM'S FUNCTION } \\
\cline { 2 - 5 } \multicolumn{2}{|c|}{ PROCEDURE } & Active & $\begin{array}{c}\text { Semi } \\
\text { Active }\end{array}$ & Passive \\
\hline \multirow{3}{*}{ Positioning } & $\begin{array}{c}\text {-Step 1- } \\
\text { CRPD } \\
\text { positioning }\end{array}$ & CRPD & & $\begin{array}{c}\text { Arms, } \\
\text { ACD }\end{array}$ \\
\cline { 2 - 5 } & $\begin{array}{c}\text {-Step 2- } \\
\text { Arms } \\
\text { positioning }\end{array}$ & Arms & CRPD & ACD \\
\hline \multirow{3}{*}{ Intervention } & $\begin{array}{c}\text {-Step 3- } \\
\text { Removal }\end{array}$ & $\begin{array}{c}\text { Arms, } \\
\text { ACD }\end{array}$ & CRPD & ACD \\
\hline \multirow{3}{*}{$\begin{array}{c}\text { Arms } \\
\text { removal }\end{array}$} & $\begin{array}{c}\text {-Step 5- } \\
\text { CRPD } \\
\text { removal }\end{array}$ & Arms & CRPD & ACD \\
\cline { 2 - 5 } & CRPD & & $\begin{array}{c}\text { Arms, } \\
\text { ACD }\end{array}$ \\
\hline
\end{tabular}

Today, the effectors are manually arranged, according to staff experience. Target of the study is to design a CRPD, able to automatically set optimal choices and to update them according to preset protocols. Thus the effectors' positions are optimally driven, to maximise effectiveness of operation site access, and minimise the intervention time.

\subsection{Choice of Architectures and Components}

A hanging robotic system, equipped with lifting devices able to lower the robot of the necessary stroke, represents an appropriate solution to adjust the robot-patient distance and to store it in the vicinity of the ceiling when not needed. For further detailing, the careful preoperative planning provides the reference information to choose the structural and functional characteristics of the CRPD. In keeping with the addressed example technologies, the study considered, both, a heavy-duty carrier, tailored as co-robotic aid for the high performance DLR arms, and a light-duty alternative, especially suited for miniature effectors. The determination of a suitable actuation structure of the CRPD is done by investigation of multiple features linked to the simultaneous presence of people (staff and patients) and equipment in the OR. The analysis is carried out using a bottom-up method that starts from the examination of the operating theatre inside the patient evolving to the area in the vicinity of the patient and ending with the exploration of the whole environment inside the OR.

According to this method, the first inquiry is how to arrange the arms' bases around the patient and, once the best relative position of the arms is established, the supporting (and positioning) device's architecture can be chosen. Several structures for the CRPD with different arms' arrangements have been evaluated in order to establish the best configuration for the arms in the patient's proximity.

The following parameters, linked to the relative position of the arms, have been optimised:
- The relative position (and distance) between the arms' bases

- The position (and distance) from each arm's base compared to the main non rotating axis

- The possibility of varying the rotational degrees

A variety of potential configurations have been evaluated using a software tool as described in [14] to establish which of them meet the arms' requirements. Doing so, typical operating theatres as shown in Fig. 5 have been investigated as well as the arms' kinematics and workspace.

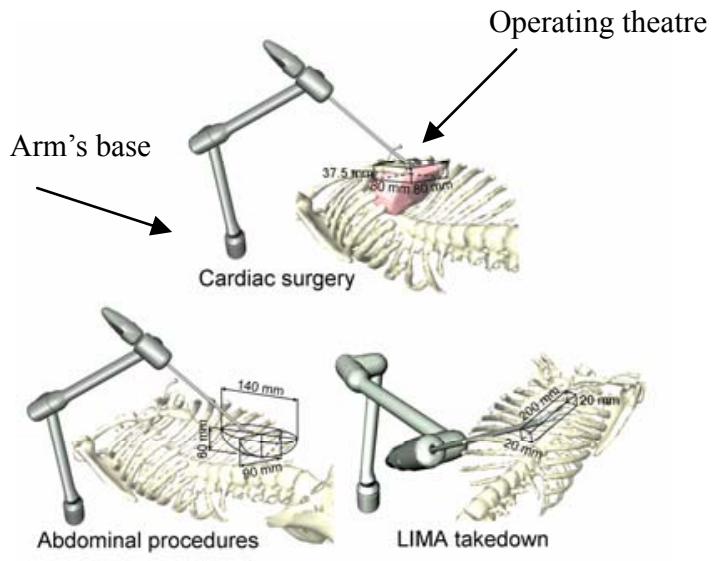

Fig. 5: Considered operating theatres.

The design alternatives have been evaluated during a simulation; the results helping to choose the design that fulfills the expected requirements in terms of:

- Reachability of the operating theatre

- Avoidance of singularities

- Avoidance of collisions

- High manipulability and accuracy

The most appropriate design alternative according to the described analysis is shown in Fig. 6. It consists of a flexible structure attached to the ceiling, with (three) arms connected to the shaft with the possibility, for each arm, to rotate according to it with independent rotational motion.

Each arm's base is mounted on an " $L$-shape connector" (see Fig. 8) that allows independent rotational motion around a main non rotating shaft. In this arrangement, the arms' bases belong to a common horizontal plane and they have a full rotational motion $\leq 360^{\circ}$ around the fixed shaft. The connectors are assumed to be hollow to contain the arms' power and signal cables that are then conducted, through the main shaft, to the power supplies arranged on the ceiling plane. The avoidance of cables on the OR's floor is a beneficial for a safer surgical environment. 


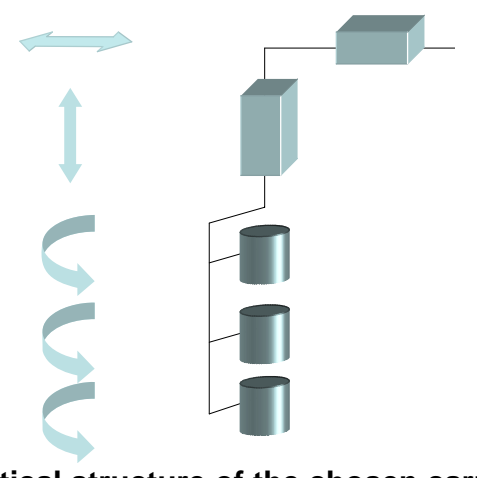

Fig. 6: Kinematical structure of the chosen carrier.

Once decided the kinematics structure of the arms, the according strokes (defining the distance of the arms from the operating theatre) have to be chosen. These are a function not only of the specific area of intervention, but also of additional aspects as for example:

- $\quad$ The estimated patient volume (base $=2200 \mathrm{~mm} \mathrm{x}$ $1200 \mathrm{~mm}$ and height $=700 \mathrm{~mm}$ )

- The usual operating table's adjustability (700/1300 $\mathrm{mm}$ )

- $\quad$ The height of the OR $(3500 / 4000 \mathrm{~mm})$

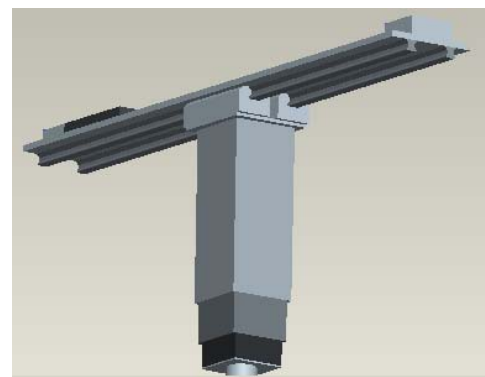

Fig. 7: Example of an exploded-view of the linear actuators of the CRPD.

The analysis provides multiple areas of best bases' positions, leading to the needed strokes concerning translation of the arms on the ceiling plane and lowering according to a vertical axis. The reference handling architecture thus looks at a hanging-down telescopic tower, running along a horizontal slide, with backlash-free stroke as shown in Fig. 7. The heavyduty arrangement, Fig. 8 exploits (three) superimposed rings, with independent rotations about the vertical axis, and the further bend/slope angular control around the transversal axes. The light-duty arrangement (see Fig. 9) carries (three) dispensers at the tower bottom, with modifiable angular hooking and with controlled bend/slope angular displacements. In both arrangements, the angular motions have limited spans, and exploit remote actuation, with motors in the distal tower part.

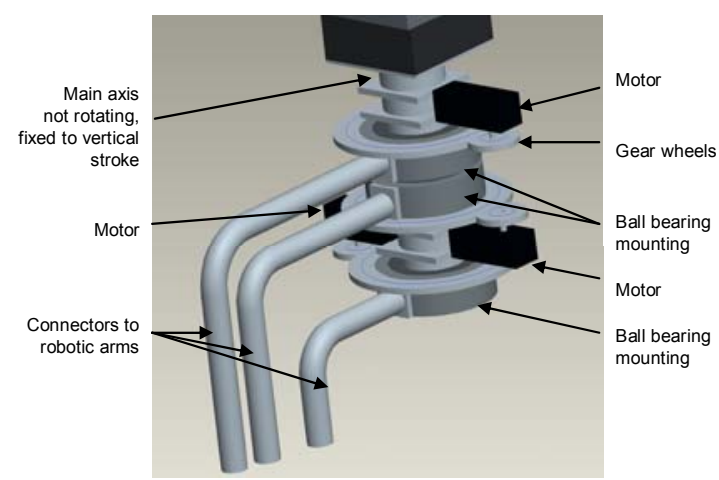

Fig. 8: Example of structural components of the heavy-duty auxiliary carrier.

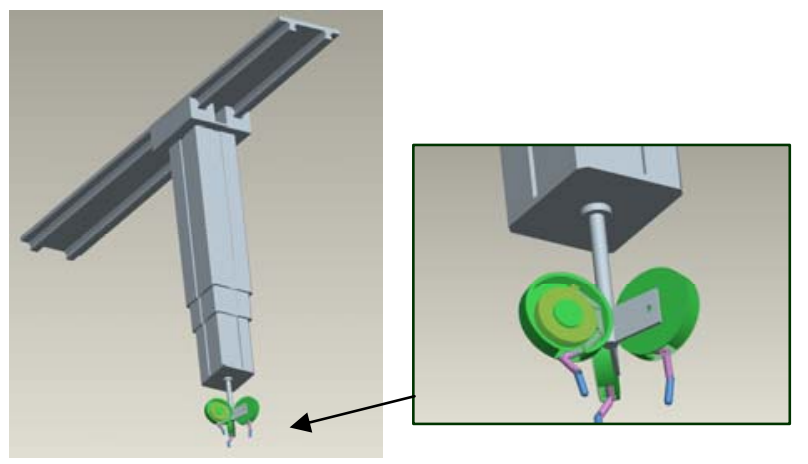

Fig. 9: Example of structural components of the light-duty auxiliary carrier.

The investigation for the CRPD concept design covered the structural assessments, to check strength and reliability properties and to evaluate geometrical tolerances and on-duty stiffness. The functional assessments basically addressed the actuation requirements, Table 2 , leaving to subsequent study, the sensors choice and the control strategies.

Table 2: Typical actuation devices for the CRPD.

\begin{tabular}{|l|c|c|c|c|}
\hline DEVICES & $\begin{array}{c}\text { Stroke } \\
(\mathrm{mm})\end{array}$ & $\begin{array}{c}\text { Dynamic } \\
\text { Forces } \\
(\mathrm{N})\end{array}$ & $\begin{array}{c}\text { Resolution } \\
(\mathrm{mm})\end{array}$ & $\begin{array}{c}\text { Speed } \\
(\mathrm{mm} / \mathrm{s})\end{array}$ \\
\hline Horizontal & 1500 & $30 \times 10^{3}$ & 0.1 & 30 \\
\hline Vertical & 700 & $4 \times 10^{3}$ & 0.1 & 11 \\
\hline Rotational (3x) & $360^{\circ}$ & 220 & 0.03 & $20 \mathrm{rpm}$ \\
\hline
\end{tabular}

\subsection{Further Design Issues}

The placement of the CRPD on the ceiling seems to solve many issues linked to the need of free space around the patient; however, the question arises as how to manage the laminar flow, necessary to maintain a sterile environment in the OR. Since during a minimally invasive procedure, the need of light is very limited, a possible solution could be to replace the lamps with the robot itself. The linear guiding table, mounted on the ceiling plane, could support both the robot and the lamps moving closer to the patient, by using an additional telescopic tower for the lamps. The robot would be lowered only during the MIRS procedure, while the lamps would be available for the rest of the time. 
Following this concept, the CRPD could even mount other mechanisms that are commonly used in the OR, e.g.: laser devices, monitoring machines (X-ray or CT machineries), insufflation equipment, and, if required, even the heart-lung machine. The initiative to mount equipment to the ceiling follows a demand currently more and more often expressed when new ORs are designed.

The attention of this study is furthermore focused on the attachment of an Automatic Changing Device for Surgical Tools (ACD-ST) to the CRPD. The changing device must not interfere with the arm's workspace, but, when needed, it has to be easily reachable by the robotic arms. The function of the changing device is to collect all the surgical instruments required from the arms for a specific operation and to provide the desired tool in the best location for the change. The optimal location for the change is clearly inside the arm's reachability.

In order to realize the procedure, the ACD-ST design is expected to have slots where to store the surgical tools and a revolving system that rotates and provides the required empty slot and the new tool. The surgeon's console will be equipped with a list of the available surgical tools (those previously inserted in the ACD-ST) from which the surgeon will be able to pick the desired instrument (or an empty spot to store the previous tool). Once the tool, the respective arm and the input command are all selected, the control system can locate the objects and the ACD-ST rotates in order to provide the instrument in the vicinity of the arm. After that, the surgeon will perform the change by either directing the arm manually towards the ACD-ST to change the tool or by simply commanding the autonomous fulfillment of the task.

In order to perform the change, the ACD-ST has also to provide a holding-tool-mechanism capable of working like e.g. a grasp. A variety of different devices have to be adopted depending on the particular connection existing between the arm and its instrument.

The control of the change mechanism represents an interesting topic that can involve different levels of sophistication in the development of a tracking system able to distinguish the different tools and the respective location. For instance, each tool could be equipped with an unique microchip that identifies (and possibly locates) it. In this context, different techniques of object-identification can be considered and implemented.

The choice of the devices must not interfere with the existing equipments in the OR; electromagnetic fields that could, for example, represent an obstacle for the accurate monitoring of the patient or to the stabilizer apparatus. Furthermore, the inserted tools have to be human compatible and to comply with the sterility standards, which is a challenging issue for the ACD-ST. With the purpose to protect the patient from potential infections, the current procedure in MIRS is to unwrap every single instrument right before it is required. With the need of preserving sterility, the ACD-ST is expected to store the instruments in appropriate dispensers. Rather than simple plastic bags, where the tools are currently stored, innovative metal casings - autoclave-compatible and automatable - are considered.
The sterility issue concerns also the robotic arms and the supporting system itself. The compatibility of materials and lubricants has to be approved according to standards as e.g. the FDA's regulations.

As the robot is in closest interaction with human, the guaranteed safety of the whole system certainly has to be taken into high consideration involving any design aspect. Concerning the CRPD, it has not only to withstand the weight of the connected devices (arms, ACD-ST, etc.), but it also has to support the accelerations of the attached devices that can be rather high; the DLR's arm, for instance, can accelerate up to $19 \mathrm{~m} / \mathrm{s}^{2}$. Even unintentional collisions between staff and robot(s) have to be taken into consideration. In this case, the stiffness of the whole system aims at preventing the TCP from dangerous, uncontrolled displacements inside the human body, and it additionally minimises the collision impact through redundancy exploitation. Thus the robot is expected to be compliant, "gentle" outside of the patient and "firm" inside.

\section{FURTHER DEVELOPMENTS AND CONCLUSIONS}

The MIRS perspectives will, most likely, modify the way the surgeons deal with the in-body interventions. Today, the technique sophistication, typically, moves along anthropocentric tracks, when the operation theatre needs to be reached by hand-carried tools and shall grant direct visual observation. Robotic surgery opens completely different options, but noteworthy outcomes do not suffer only technology gaps, as, first of all, sophistication should encompass relevant paradigm shifts in the operation protocols. This means a rather different approach in the design of the robotic instrumental aids, and the present investigation has been facing quite relevant issues, combining the minimally invasive robotic surgery technique with the split-duty idea. For concreteness, the research preferred continuous feasibility checks, thus, the co-robotic means and planning deal with existing developments: the high performance modular DLR arms and the (some slightly futuristic) miniature probes. This way, both, actual and on-progress options are considered, bringing forward the procedural innovation as preliminary step of the instrumental updating.

On these assumptions, the functional versatility of the corobotic equipment ought to be explored, linked to the capabilities of the front effectors; the enhanced performance can be enabled on-progress, depending on the articulated probe/arm advances. Typically, for instance, the constraint on mobility aims at making given achievements feasible, disregarding the (more or less relevant) side-effects. Invasiveness is lowered, but not avoided, when vital organs are involved. Now, an articulated in-body probe has better dexterity with blocks higher in number and smaller in size; cable-actuation quickly faces unavoidable drawbacks, due to force-coupling among blocks. Happily, the current microelectro-mechanical-systems, mems, technology provides effective means to obtain proper arrangements, and future devices will, most likely, quickly assure improved performance. In any case, the split-duty idea permits conceiving auxiliary carriers fitted out for evolving end-effectors, as soon as these 
appear. This suggests parallel investigation of both reference technologies.

\section{ACKNOWLEDGMENT}

The work is done within the $\mathrm{PhD}$ investigation of the coauthor Silvia Frumento. The research periods at the DLR (Weßling, Institute of Robotics and Mechatronics) supervised by Prof. Gerd Hirzinger, and at the University of Hawaii at Manoa (Honolulu, Dept. Mechanical Engineering) supervised by Prof. Yuling Yan, are gratefully acknowledged, as well as the fellowships of CARIGE Foundation and MIUR Vigoni, which supported the travels and stays.

\section{REFERENCES}

[1] Cepolina, F., and Michelini, R. C., 2004, "Robots in medicine: a survey of in-body nursing aids. Introductory overview and concept design hints", $35^{\text {th }}$ Intl. Symposium on Robotics, ISR 2004, Paris.

[2] Cepolina, F., and Michelini, R. C., 2004, "Review of robotic fixtures for minimal-invasiveness surgery", Intl. J. Medical Robotics \& Computer-Assisted Surgery, 1(1), pp. 43-63.

[3] Taylor, R. H., and Stoianovici D., 2003, "Medical Robotics in Computer-Integrated Surgery", IEEE Transactions on Robotics and Automation, 19(5).

[4] Ortmaier, T., Seibold, U., Hagn, U., Böhm, D. H, Reichenspurner, H., and Hirzinger, G., 2001, "Autonomy and Haptic Feedback in Minimally Invasive Robotic Surgery", in Fourth Annual Meeting of the International Society for Minimally Invasive Cardiac Surgery (ISMICS).

[5] Falk, V., Diegeler, A., Walther, T., Löscher, N., Vogel, B., Ulmann, C., Rauch, T., and Mohr, F. W., 1999, "Endoscopic Coronary Artery Bypass Grafting on the Beating Heart Using a Computer Enhanced Telemanipulation System", Heart Surg. Forum, 2, pp. 199 205.
[6] Seibold, U., Kübler, B., and Hirzinger, G., 2005, "Prototype of Instrument for Minimally Invasive Surgery with 6-Axis Force Sensing Capability", ICRA 2005 IEEE International Conference on Robotics and Automation, Barcelona, Spain.

[7] Cepolina, F., 2005, "Development of micro-tools for surgical applications", Università di Genova, Italy \& Université P.\&M. Curie, Paris, PhD Thesis.

[8] Frumento, S., Michelini, R.C, 2004, "Progetto di ausiliari co-robotici in tele-chirurgia", Convegno ADM-AIAS: Innovazione nella progettazione industriale, Bari, ISBN 88900637-2-6, p. 351

[9] Cepolina, F., Michelini, R.C., 2003, "A family of corobotic surgical set-ups", Intl. J. Industrial Robots, ISSN 0143991 X, 30(6), pp. 564-574.

[10]Cepolina, F., Michelini, R.C., 2004, "Progetto di dispositivi di robotica chirurgica", Convegno ADM-AIAS: Innovazione nella progettazione industriale, Bari, ISBN 88900637-2-6, p. 341.

[11] Ortmaier, T., Weiss, H., Hagn, U., Grebenstein, M., Nickl, M., Albu-Schaeffer, A., Ott, C., Joerg, S., Konietschke, R., Hirzinger, G., Essenreiter, R., and Bertram, M., 2005, "Ein neuer Roboter zum navigierten Setzen von Pedikelschrauben" (in German), VDI Wissensforum Mechatronik, Deutschland.

[12] Konietschke, R., Weiss, H., Ortmaier, T., and Hirzinger, G., 2004, "A Preoperative Planning Procedure for Robotically Assisted Minimally Invasive Interventions", conference CURAC, Germany.

[13] Ortmaier, T., 2004, "Motion Compensation in Minimally Invasive Robotic Surgery", Technical University of Munich, PhD Thesis.

[14] Konietschke, R., Ortmaier, T., Weiss, H., Engelke, R., and Hirzinger, G., 2003, "Optimal Design of a Medical Robot for Minimally Invasive Surgery", conference CURAC, Germany. 